\title{
Malaria por Plasmodium vivax: curación del ataque agudo con tres dosis diferentes de primaquina y dosis fija de cloroquina. Antioquia, Colombia, 2003-2004
}

\author{
Jaime Carmona-Fonseca, Gonzalo Álvarez, Silvia Blair \\ Grupo de Malaria, Universidad de Antioquia, Medellín, Colombia.
}

Introducción. Cloroquina $(\mathrm{CQ})$ y primaquina $(\mathrm{PQ})$ se usan en Colombia para tratar la malaria vivax no complicada, pero son escasas las evaluaciones de su eficacia.

Objetivos. a) investigar la clínica de la malaria vivax en dos zonas con muy diferente nivel endémico; b) evaluar la respuesta terapéutica antimalárica obtenida con el tratamiento estándar $C Q-P Q$ tomadas simultáneamente; c) comparar la eficacia del tratamiento estándar y la de otros dos esquemas que conservan la dosis total de CQ pero varían la de $P Q$; d) identificar los efectos adversos ocurridos durante el tratamiento CQ-PQ.

Materiales y métodos. Diseño aleatorio, controlado, no ciego; tres grupos definidos según dosis total de PQ: 45, 105 y $210 \mathrm{mg}$. Todos recibieron CQ (1500 mg, en 48 horas) y simultáneamente PQ. Pacientes captados consecutivamente, en Turbo y El Bagre. Seguimiento durante 28 días. La respuesta terapéutica antimalárica clasificada en falla precoz, falla tardía y respuesta adecuada.

Resultados. Se trataron 228 pacientes y la respuesta terapéutica antimalárica se evaluó en 210. El cuadro clínico encontrado es similar al descrito por otros autores. En todos los tres grupos, la parasitemia se eliminó en 24 horas de tratamiento con CQ en $11 \%$ de los pacientes, en 48 horas en $66 \%$ y en 72 horas en $97 \%$. Todos los 210 pacientes respondieron adecuadamente al tratamiento CQ-PQ. La dosis variable de PQ no afectó esta respuesta.

Conclusiones. CQ-PQ debe conservarse como la primera opción terapéutica y debe evaluarse en otras áreas. Se propone la administración concomitante de CQ y PQ.

Palabras clave: Plasmodium vivax, malaria, cloroquina, primaquina, eficacia, ensayo clínico controlado aleatorio [Tipo de Publicación].

Plasmodium vivax malaria: treatment of primary attacks with primaquine, in three different doses, and a fixed dose of chloroquine, Antioquia, Colombia, 2003-2004

Introduction. Chloroquine (CQ) and primaquine (PQ) are used in Colombia for treatment of uncomplicated vivax malaria but there are few efficacy studies of this scheme.

Objectives. a) to investigate the clinical picture of vivax malaria in adult patients; $b$ ) to evaluate the antimalarial treatment response to the standard scheme $C Q-P Q ; c)$ to compare the efficacy of the standard scheme and two alternative schemes with equal dose of $C Q$ and different $P Q$ dose; d) to identify the adverse effects of $C Q-P Q$ treatment.

Materials and methods. Randomized, nonblind design; three groups, defined according to total dose of PQ: 45, 105 and $210 \mathrm{mg}$. All patients received CQ (1500 mg, in 48 hours), which was followed by PQ. Patients were recruited in Turbo and El Bagre. The follow-up period was 28 days. Antimalarial treatment response was classified according to the WHO-2001 protocol (early failure, late failure, adequate response).

Results. In total 228 patients were recruited, 18 were lost between days 4 and 27; the antimalarial treatment response was evaluated in 210 . The clinical findings were similar to those described by other authors. Parasitaemia clearance was confirmed during the first 24 hours of CQ treatment in $11 \%$ of patients. At 48 hours $66 \%$ and at 72 hours $97 \%$ of patients were negative. All patients (210) displayed adequate treatment response to the CQ-PQ treatment. The different doses of $P Q$ did not affect this response. 
Conclusions. CQ-PQ should be conserved as the first treatment choice and should be evaluated in other areas. Simultaneous administration of $C Q$ and $P Q$ is proposed.

Keywords: Plasmodium vivax, malaria, chloroquine, primaquine, efficacy, randomized controlled trial [Publication Type].

La malaria causada por Plasmodium vivax es la más frecuente en Colombia (65 a $70 \%$ ) y en América (alrededor de $80 \%$ ). En las zonas de Urabá y el Bajo Cauca se presenta el $90 \%$ del paludismo en Antioquia y $P$. vivax aporta 65 a $70 \%$ del total $(1,2)$.

Las manifestaciones clínicas de la malaria por $P$. vivax no complicada se han estudiado en Urabá y el Bajo Cauca antioqueño (3-5). En pacientes hospitalizados por malaria en Medellín también exploramos la clínica del paludismo por $P$. vivax comparado con el causado por Plasmodium falciparum (6).

Desde hace más de 50 años, el tratamiento de la malaria por $P$. vivax no complicada se hace en Colombia con cloroquina y primaquina (CQ-PQ) como primera opción $(7,8)$. Aparte de ese tratamiento estándar $(7,8)$, existen otras alternativas que modifican los $\mathrm{mg} / \mathrm{kg}$ de peso, los días de uso de la $P Q$ o ambos (9-14). El tratamiento estándar de la malaria por $P$. vivax con CQ sirve para eliminar tanto las formas asexuales sanguíneas como los gametocitos, pero no los hipnozoítos, que requieren la $P Q(7,15)$. Hay sinergismo entre $C Q$ o quinina con $P Q$ para prevenir recaídas de malaria por $P$. vivax $(16,17)$. El protocolo para evaluar la respuesta terapéutica antimalárica en paludismo por $P$. vivax implica el suministro de CQ $(25 \mathrm{mg} / \mathrm{kg}$ en tres días, o sea 48 horas), seguido de 28 días de observación con evaluaciones clínicas y parasitarias (18); esa evaluación de la eficacia de la $C Q$ excluye el suministro de $P Q$ en esos 28 días.

$P$. vivax ha permanecido sensible a la $C Q$ en casi todas las zonas del mundo malárico, y sus formas sanguíneas se eliminan con concentraciones de

\footnotetext{
$\overline{\text { Correspondencia: }}$

Jaime Carmona-Fonseca, Calle 62 No. 52-59, laboratorio 610, Medellín, Colombia.

Telefax: (574) 2106487.

jaimecarmonaf@hotmail.com

Recibido: 06/03/06; aceptado: 24/07/06
}

$\mathrm{CQ}$ en suero o plasma de 30 y $15 \mathrm{ng} / \mathrm{mL}$ (19), respectivamente. Sin embargo, en 1989 se hizo la primera declaración de "malaria vivax cloroquino-resistente" en Papua Nueva Guinea, y desde entonces se han sumado informes similares en ese mismo lugar y en la vecina Indonesia y, con menos frecuencia, en Myanmar, Tailandia, Borneo, India (20), Brasil $(21,22)$, Guyana (23), Colombia (9) y Perú (24).

Es fundamental precisar qué se entiende por malaria por $P$. vivax resistente a CQ. Consideramos, con Ruebush y colaboradores (2003) (24), que deben definirse criterios claros y precisos para aceptar una infección por $P$. vivax como resistente a cloroquina; ellos proponen tres criterios: 1 ) haber recibido completamente, en la forma preestablecida y con supervisión adecuada, el tratamiento estándar con $\mathrm{CQ}$; 2) la recurrencia de la parasitemia debe ocurrir dentro de los 28 días de seguimiento contados a partir del inicio del tratamiento; 3) en el momento de la recrudescencia, los niveles de CQ y de desetil$C Q$ en sangre deben ser mayores de 90 a $100 \mathrm{ng} /$ $\mathrm{mL}$. En Suramérica, solamente dos informes sobre falla satisfacen tales criterios: el de un viajero de Guyana, en 1996 (23), y el de dos pacientes de la Amazonia peruana, en 2003 (24).

En relación con la falla terapéutica antimalárica se requiere precisar el significado de los términos recurrencia, recrudescencia, recaída y reinfección (25-29). Se considera que en la malaria por $P$. vivax (25) la recurrencia de la parasitemia en algún momento de los 28 días de seguimiento posterior a la administración efectiva del tratamiento estándar con $C Q$ demuestra que existe resistencia de $P$. vivax a la CQ. Si la recurrencia sucede antes del día 17 es casi seguro que se trata de una recrudescencia. Si la recurrencia sucede entre los días 17 a 28 se trata de recrudescencia o de una recaída por parásitos resistentes a cloroquina. Si la recurrencia sucede después del día 28, puede tratarse de una recaída por parásitos sensibles a CQ. 
En Turbo, la combinación estándar de $C Q$ y $P Q$ para tratar la malaria por $P$. vivax no complicada mostró eficacia de $100 \%$ para curar el cuadro agudo de paludismo (30) siguiendo el protocolo de la Organización Mundial de la Salud (OMS) (18). Conocemos un informe que relata la aparición de tres casos de falla terapéutica entre 27 soldados con malaria por $P$. vivax tratados con el esquema estándar de $C Q$ y $P Q$, es decir falla de $11 \%$ (9); esos soldados recibieron sólo una dosis total de $1.500 \mathrm{mg}$ de $\mathrm{CQ}$ (no tomaron PQ).

Esos antecedentes motivaron la realización de la presente investigación, cuyos objetivos fueron: a) investigar la clínica de la malaria por $P$. vivax en dos zonas con muy diferente nivel endémico; b) evaluar la respuesta terapéutica antimalárica obtenida con el tratamiento estándar de CQ y PQ tomadas simultáneamente; c) comparar la eficacia del tratamiento estándar y la de otros dos esquemas que conservan la dosis total de $C Q$, pero varían la de $P Q$; d) identificar los efectos adversos ocurridos durante el tratamiento con $\mathrm{CQ}$ y $P Q$.

\section{Materiales y métodos}

Los sitios del estudio fueron Turbo (Urabá) y EI Bagre (Bajo Cauca).

En tres artículos recientes hemos definido el contexto para interpretar la falla y la resistencia antimaláricas en las zonas de Urabá y Bajo Cauca en el departamento de Antioquia, Colombia $(1,2,31)$. Urabá y el Bajo Cauca son las dos zonas palúdicas más importantes del departamento de Antioquia y aportan $90 \%$ del paludismo en este departamento. En Turbo $\left(8^{\circ} 05^{\prime} 42^{\prime \prime} \mathrm{N}, 76^{\circ} 44^{\prime}\right.$ $\left.123^{\prime \prime}\right), 60 \%$ de la población se encuentra dispersa en la zona rural (aldeas o pequeños poblados), mientras en El Bagre $\left(7^{\circ} 35^{\prime} 25^{\prime \prime} \mathrm{N}, 74^{\circ} 48^{\prime} 27^{\prime \prime}\right)$ sólo $38 \%$ es rural. La estructura de edad de las dos poblaciones es bastante similar $(1,2)$. En los últimos 50 años, y sobre todo en los últimos veinte, las migraciones por razones económicas o sociopolíticas han tenido fuerte expresión en Urabá y el Bajo Cauca. Estas migraciones no han sido estudiadas en forma adecuada, pero es muy probable que hayan contribuido a acentuar el problema malárico y a diseminar clones de $P$. falciparum resistentes a cloroquina y otros medicamentos, tal como sucede en la frontera de Tailandia con Camboya y Myanmar (32).

La principal actividad económica del Bajo Cauca es la minería de aluvión, seguida por la ganadería $y$, en menor grado, los cultivos de arroz y sorgo. La agroindustria bananera es la principal actividad de Urabá; la ganadería es la segunda actividad económica; la explotación de madera, a partir de la tala de selvas naturales, es la tercera actividad de esa región. La participación de la población económicamente activa de las dos regiones según los sectores económicos fue muy similar en 1998: primario $49 \%$, secundario $5 \%$ y terciario $46 \%(1,2)$.

La mediana del índice parasitario anual (IPA) y del número de casos de paludismo entre 1998 y 2004 fue de 43,98 por mil en Urabá (19.953 casos), de 47,46 por mil en Turbo (5.531 casos), de 72,49 en el Bajo Cauca (15.665 casos) y 85,32 en El Bagre (4.896 casos) (con base en datos de la Dirección Seccional de Salud de Antioquia, DSSA).

\section{Clase de estudio}

El diseño correspondió a un estudio clínico controlado, balanceado, no ciego, en el que se captaron sucesivamente 228 pacientes con malaria debida sólo a $P$. vivax diagnosticada por gota gruesa, quienes se asignaron al azar (mediante balotas marcadas con el grupo) a uno de los tres tratamientos según la dosis total de $P Q$ (3 días $=45 \mathrm{mg}, 7$ días $=105 \mathrm{mg}$, y 14 días = $210 \mathrm{mg}$ ).

\section{Población de referencia}

La población de referencia la constituyeron los pacientes adultos (mayores de 18 años), hombres y mujeres residentes tanto en el área urbana como rural de Turbo o El Bagre, afectados con malaria por $P$. vivax.

\section{Diseño de la muestra}

La muestra se diseñó con criterios estadísticos y epidemiológicos con el fin de comparar la proporción de fallas terapéuticas entre los dos grupos extremos (14 días con $210 \mathrm{mg}$ de dosis total de $P Q$ y tres días con $45 \mathrm{mg}$ de $\mathrm{PQ}$ ). Se partió de las siguientes premisas: a) el tratamiento estándar produce curación en $100 \%$ de los 
pacientes (que debe tratarse como 99\% para poder hacer las operaciones estadísticas); b) hay un informe colombiano (9) que refirió una falla de $11 \%$, y que nosotros redondeamos a $15 \%$; c) esto lleva a decir que se espera $99 \%$ de respuesta adecuada en el grupo con 14 días y $84 \%$ (99 menos $15 \%$ ) en el grupo con tres días. El nivel de significación (alfa) se tomó en 0,05 (que corresponde a $Z=1,96$ ) y el error beta en 0,20 . Se aplicó esta ecuación con el programa Epidat 3:

$\mathrm{n}=[(\mathrm{A}+\mathrm{B}) / \mathrm{C}] 2$, donde:

$A=Z 1-a / 2$ con $=(p 1+p 2) / 2$

$B=Z 1-B$

$\mathrm{C}=(p 2-p 1)$

Es decir:

$A=1,96(1$ a 0,25$)$, dado que $=(0,99+0,84) / 2=$ 0,915

$B=1,96(1$ a 0,20$)$

$\mathrm{C}=(0,99-0,84)$

El tamaño muestral resultó en 54 pacientes por grupo, incrementado a 70 en aras de compensar posibles pérdidas durante el seguimiento, para un total de 210 pacientes en el estudio.

La captación de los pacientes se hizo en forma pasiva cuando llegaban por su propia iniciativa al puesto de malaria del área urbana de Turbo o de El Bagre. Se captaron pacientes durante 13 meses ( 4 septiembre de 2003 hasta 15 septiembre de 2004). Se asignaron en forma aleatoria (balotas con el nombre del tratamiento) a cada grupo y fueron evaluados durante 28 días.

\section{Criterios de inclusión}

1. Malaria exclusivamente por $P$. vivax según el examen parasitológico de sangre.

2. No tener malaria grave (complicada).

3. Tener una parasitemia mínima de un parásito por cada 6 a 8 glóbulos blancos, lo que corresponde aproximadamente a 1.000 parásitos $/ \mu \mathrm{L}$.

4. Ser mayor de 14 años.

5. No estar en embarazo según la prueba inmunológica en tirilla disponible en el mercado.
6. Aceptar participar en el estudio y firmar el consentimiento informado.

\section{Criterios de exclusión}

1. Incumplir algún control en cualquiera de los tres días iniciales.

2. Negarse a tomar los medicamentos.

3. Consumir otro medicamento antipalúdico.

4. Retirar el consentimiento informado.

\section{Diagnóstico de malaria}

El diagnóstico parasitario lo hizo un laboratorista profesional con entrenamiento de varios años en malaria mediante gota gruesa y extendido de sangre periférica coloreadas con Field y Giemsa, respectivamente. El recuento de la parasitemia se hizo contando el número de formas asexuadas en 200 glóbulos blancos según los criterios de la OMS-Organización Panamericana de la Salud (OPS) (33).

\section{Evaluación médica y tratamiento de malaria}

Un médico general, con amplia experiencia en el diagnóstico y tratamiento de la malaria, evaluó a cada paciente que ingresó al estudio, hizo una evaluación clínica inicial y durante los días de seguimiento 1 (24 horas de tratamiento), 2 (48 horas de tratamiento), 3 (72 horas de tratamiento, es decir 24 horas después de haber terminado la $C Q$ ), y en el día 28.

Se citó a todos los pacientes, independientemente del grupo al que se asignaron al azar, al puesto de malaria para suministrarles el tratamiento (CQ$P Q$ ) en cada uno de los tres primeros días, mientras que para suministrar la $P Q$ en los días siguientes se hizo una búsqueda activa de los pacientes en su lugar de residencia para garantizar que tomaran el medicamento. Los fármacos fueron suministrados por la Dirección Seccional de Salud de Antioquia (DSSA), así: cloroquina, lote C19081-0804 y primaquina, lote 070902-0905, y se administraron así: $1.500 \mathrm{mg}$ de CQ para cada paciente en 48 horas (600 mg día uno, $450 \mathrm{mg}$ día dos y $450 \mathrm{mg}$ día tres, siempre con 24 horas de intervalo entre una y otra dosis). Simultáneamente con la toma de $C Q$, el paciente tomó $P Q$ según el grupo de tratamiento asignado (45, 
105 y $210 \mathrm{mg})$. Los medicamentos se administraron con 150 a $200 \mathrm{~mL}$ de agua potable Bajo estricta supervisión del equipo de investigadores.

Los pacientes que completaron 28 días sin síntomas o signos palúdicos y sin parasitemia participaron desde el día 29 en un proyecto para evaluar la eficacia de la PQ contra las recurrencias de malaria por $P$. vivaxy, por tanto, los integrantes de los grupos que recibieron 45 y $105 \mathrm{mg}$ de $P Q$ en la etapa inicial no tomaron más este medicamento.

\section{Definición del efecto: respuesta terapéutica antimalárica}

La curación del ataque malárico se midió con criterios clínicos (desaparición de los síntomas y los signos hallados en la evaluación de ingreso) y parasitarios (eliminación de la parasitemia según gota gruesa y extendido de sangre). La curación del paludismo se evaluó durante una fase de seguimiento de 28 días, al final de la cual se consideraba a la persona curada si no había manifestaciones clínicas ni parasitemia. La presencia de manifestaciones clínicas, de parasitemia o de ambas implicó la declaración de no curado.

Para evaluar esta respuesta terapéutica antimalárica se aplicó el protocolo de la "Red de Farmacovigilancia en Malaria en Colombia. Evaluación in vivo" (septiembre 2002), desarrollado por investigadores del Cideim (Cali, Colombia) y del Grupo de Malaria de la Universidad de Antioquia (Medellín, Colombia), para evaluar la respuesta terapéutica antimalárica tanto en la malaria por $P$. falciparum como por $P$. vivax; este protocolo es una adaptación del protocolo "Evaluación de la eficacia terapéutica de los medicamentos para el tratamiento del paludismo por Plasmodium falciparum sin complicaciones en las Américas", creado por OMS-OPS (marzo 1998) $(34,35)$. El protocolo del Grupo de Malaria y el CIDEIM concuerda, en esencia, con el protocolo (borrador) de la OMS para evaluar la respuesta terapéutica antimalárica en malaria por P. vivax (18). La respuesta terapéutica antimalárica de los pacientes al tratamiento de CQ y PQ se clasifica en falla precoz (la detectada hasta 24 horas después de terminar la toma de CQ), falla tardía (la que ocurre posteriormente y hasta el día 28) y respuesta adecuada (exitosa). Para declarar la falla tardía se requirió siempre la presencia de parásitos en la gota gruesa de sangre periférica acompañados o no de síntomas o signos clínicos $(18,34,35)$.

\section{Controles clínicos y parasitarios}

Los días 1, 2, 3 y 28 se hicieron evaluaciones médicas y con gota gruesa. Cabe advertir que el protocolo OMS-2001 también hace evaluaciones los días 14 y 21 , que nosotros no efectuamos.

\section{Efecto adverso}

Se hizo especial énfasis en detectar todo efecto adverso que apareciera en el seguimiento (28 días) de la respuesta terapéutica antimalárica. Concebimos la evaluación de los efectos adversos como parte ineludible del examen de la respuesta terapéutica antimalárica, que no debe limitarse a averiguar si el tratamiento curó o no la enfermedad, sino que debe indagar por los problemas que el tratamiento generó. Es conveniente diseñar y poner en operación un sistema de vigilancia epidemiológica de la toxicidad de los antipalúdicos al tiempo que se evalúa su eficacia; como otros han señalado (36), el conocimiento de estos problemas es incipiente en Colombia y en muchos otros lugares de endemia palúdica.

Por efecto adverso se entendió cualquier síntoma o signo clínico nuevo, o el agravamiento de uno existente, ocurrido durante el período de tratamiento del paciente (definición del protocolo Grupo de Malaria y el CIDEIM ) y limitado a los 28 días iniciales (tres con CQ y luego hasta 14 días con $P Q$ ).

De acuerdo con el protocolo Grupo de Malaria y el CIDEIM, se evaluó la relación entre el efecto adverso y el medicamento mediante la siguiente escala: no se sabe, ninguna relación, posible, poco probable, probable, definitiva. El resultado del efecto adverso se clasificó en recuperación completa, todavía presente (al terminar el seguimiento), secuelas, muerte, no se sabe. La conducta aplicada por el médico tratante se clasificó en ninguna, suspensión del tratamiento, 
hospitalización, otra. Se preguntó específicamente si el efecto adverso se consideraba serio, lo que se definió como cualquier manifestación clínica que resultase en la muerte del paciente, que amenazase su vida, que obligase a hospitalizar al enfermo, que produjese incapacidad o discapacidad significativa o permanente, que pudiese ser peligroso de cualquier manera para el paciente, o que requiriese intervención para prevenir los resultados antes referidos.

\section{Aspectos éticos}

Cada paciente fue personalmente informado de la investigación, tanto en forma verbal como escrita; se le explicaron las alternativas terapéuticas que tenía, de las cuales una de ellas era el tratamiento aplicado en forma rutinaria (tratamiento durante 14 días); si aceptaba participar debía firmar ante testigos el documento de consentimiento informado, el cual, junto con el proyecto en su conjunto, había sido aprobado por el comité de ética del Centro de Investigaciones Médicas de la Facultad de Medicina de la Universidad de Antioquia.

\section{Análisis de la información}

Se creó una base de datos relacional con el programa Epilnfo 6.04. La información fue captada en el terreno por miembros del equipo de investigación y fue ingresada en la base de datos por otro de los investigadores. El análisis de la información se hizo con los programas SPSS versión 10.0 para Windows y Epilnfo 6.04 para D.O.S. El análisis estadístico consistió en calcular porcentajes, medidas de tendencia central y dispersión, y evaluar la asociación entre las variables cualitativas mediante la prueba de ji al cuadrado $\left(\mathrm{X}^{2}\right)$. La comparación de promedios se hizo con la prueba $t$ de Student o la prueba no paramétrica de Mann-Whitney.

\section{Resultados}

Se captaron y trataron 228 pacientes, de los cuales 18 se perdieron antes de 28 días; en los 210 restantes se pudo verificar la respuesta terapéutica. Las características demográficas de los 210 pacientes indican que eran predominantemente mestizos, masculinos, con promedio de edad de 30 años y $61 \mathrm{~kg}$ de peso corporal; 38\% tenía antecedentes de malaria en el año previo y 2,4 episodios de malaria en el mismo periodo; tenían en promedio 5 días de evolución de la enfermedad y presentaban parasitemia promedio de 6.000 parásitos/ $\mu \mathrm{L}$ (cuadro 1).

\section{Cuadro clínico}

Los síntomas más frecuentes encontrados en los pacientes el día de ingreso al estudio se resumen en el cuadro 2. Las mayores frecuencias correspondieron a los síntomas clásicos del

Cuadro 1. Características de los pacientes con evaluación de la respuesta terapéutica según el municipio de residencia.

\begin{tabular}{|c|c|c|c|c|}
\hline Variables & $\begin{array}{c}\text { Turbo } \\
n=138 ;(\%)\end{array}$ & $\begin{array}{c}\text { El Bagre } \\
n=72 ;(\%\end{array}$ & $\begin{array}{c}\text { Total } \\
n=210 ;(\%)\end{array}$ & Valor $p^{(1}$ \\
\hline Sexo masculino & $89(64,0)$ & $53(72,6)$ & $142(67,0)$ & 0,181 \\
\hline Etnia "mestiza" (2) & $129(92,8)$ & $66(90,4)$ & $195(92,0)$ & 0,629 \\
\hline Antecedentes de malaria ${ }^{(3)}$ & $42(32,6)$ & $27(43,5)$ & $69(36,1)$ & 0,302 \\
\hline Edad (años) & $31,2 \pm 13,8$ & $27,9 \pm 10,4$ & $30,1 \pm 12,8$ & 0,078 \\
\hline Peso corporal $(\mathrm{kg})$ & $61,2 \pm 8,7$ & $62,1 \pm 9,7$ & $61,5 \pm 9,1$ & 0,487 \\
\hline Número episodios palúdicos ${ }^{(3)}$ & $1,8 \pm 1,4$ & $2,9 \pm 2,3$ & $2,2 \pm 1,9$ & 0,008 \\
\hline \multicolumn{5}{|l|}{ Días evolución enfermedad } \\
\hline Enfermedad & $5,0 \pm 4,0$ & $4,5 \pm 3,0$ & $4,8 \pm 3,7$ & 0,391 \\
\hline Temperatura ${ }^{\circ} \mathrm{C}$ & $37,4 \pm 1,4$ & $37,2 \pm 1,2$ & $37,3 \pm 1,3$ & 0,700 \\
\hline Parásitos asexuales $/ \mu \mathrm{L}$ & $6400 \pm 6761$ & $5096 \pm 5390$ & $5955 \pm 6344$ & 0,157 \\
\hline Gametocitos/ $\mu \mathrm{L}$ & $503 \pm 100$ & No medidos & $503 \pm 100$ & No aplica \\
\hline
\end{tabular}

(1) Valor de probabilidad ( $p$ ) para las pruebas de ji al cuadrado $\left(\mathrm{X}^{2}\right)$ o de Mann-Whitney entre los datos de Turbo y El Bagre. Sólo hay diferencia estadísticamente significativa en los valores de "número de episodios de malaria en el año previo", que es mayor en El Bagre.

(2) "Mestizo": cualquier mezcla entre "blanco", "negro" e "indio" y sus descendientes.

(3) Antecedentes palúdicos y número de episodios en el año anterior. 
Cuadro 2. Síntomas más frecuentes narrados en la enfermedad actual.

\begin{tabular}{lrrr}
\hline Síntoma & \multicolumn{3}{c}{ Total $\mathbf{n}=\mathbf{2 1 0}$} \\
\cline { 2 - 4 } & $\mathbf{n}^{(1)}$ & $\%$ & $\mathbf{p}^{(2)}$ \\
\hline Cefalea & 187 & 88,2 & 0,000 \\
Dolor osteomuscular & 174 & 82,1 & 0,005 \\
Fiebre & 148 & 69,8 & 0,000 \\
Anorexia & 135 & 63,7 & 0,000 \\
Escalofrío & 134 & 63,2 & 0,000 \\
Sudoración & 113 & 53,3 & 0,000 \\
Náusea & 91 & 42,9 & 0,000 \\
Dolor abdominal & 75 & 35,4 & 0,030 \\
Tos & 64 & 30,2 & 0,586 \\
Vómito & 33 & 15,6 & 0,000 \\
Disnea & 26 & 12,3 & 0,005 \\
Odinofagia & 23 & 10,8 & 0,000 \\
Diarrea & & 10,8 & 0,056 \\
Prurito & 9 & 4,2 & 0,700 \\
Brote en piel & 7 & 3,3 & 0,314 \\
\hline
\end{tabular}

(1) Los valores se refieren al número de personas con determinado síntoma y al porcentaje que representan con respecto a 210 .

(2) Valor de probabilidad de ji al cuadrado (Pearson) para comparar la frecuencia entre El Bagre y Turbo. Siempre que $p<0,05$, la frecuencia del síntoma fue significativamente mayor en El Bagre.

paludismo (cefalea, dolor osteomuscular, fiebre, anorexia, escalofrío, entre otros). La frecuencia de síntomas fue estadísticamente mayor en El Bagre que en Turbo.

Los signos más frecuentemente hallados por el médico en el momento de ingreso al estudio fueron, en su orden, palidez conjuntival, dolor al palpar el abdomen, hepatomegalia y esplenomegalia (cuadro 3). Ninguno de los siguientes signos se presentaba en los pacientes: sangrado, cianosis, ictericia, petequias, equimosis, edema, crépitos, roncos, estertores, soplo cardíaco, desorientación témporo-espacial, delirio, trastorno de la conducta, convulsiones, pupilas anormales.

\section{Respuesta terapéutica antimalárica a $C Q$ y $P Q$}

De los 228 pacientes captados, 18 (8\%) se perdieron antes de completar 28 días de seguimiento, por lo cual no se pudo establecer la respuesta terapéutica. En los 210 enfermos en quienes se determinó el efecto (respuesta terapéutica antimalárica), se comprobó curación total (clínica y parasitaria) del paludismo agudo en $100 \%$ de los casos con cualquiera de las tres
Cuadro 3. Signos clínicos hallados por el médico en el momento de ingreso del paciente.

\begin{tabular}{llrc}
\hline Área & \multicolumn{1}{c}{ Signo } & $\begin{array}{r}\text { Total } \\
\mathbf{n}{ }^{(1)}\end{array}$ & $\begin{array}{r}\mathbf{n = 2 1 0} \\
\%\end{array}$ \\
\hline Piel & Palidez conjuntival & 89 & 42,0 \\
& Palidez palmar & 6 & 2,8 \\
& Prurito & 9 & 4,2 \\
\multirow{4}{*}{ Abdomen } & Brote en piel & 7 & 3,3 \\
& Dolor abdomen al palpar & 75 & 35,4 \\
& Hepatomegalia $(1-3 \mathrm{~cm})$ & 37 & 17,5 \\
\multirow{4}{*}{ Otras } & Esplenomegalia $(1-4 \mathrm{~cm})$ & 23 & 10,8 \\
& Odinofagia & 23 & 10,8 \\
& Coluria & 12 & 5,7 \\
& Sibilancias & 2 & 0,9 \\
\hline
\end{tabular}

(1) Los valores se refieren al número de personas con determinado síntoma.

dosis totales de PQ. Los siete pacientes con parasitemia a las 24 horas de finalizar la administración de $C Q$ no son fallas terapéuticas según el protocolo de la OMS porque los niveles de parasitemia en cada sujeto son menores de $1 \%$ de los hallados en el momento del ingreso; además, en el día 28 esos sujetos tenían gota gruesa negativa.

La evolución temporal de los signos vitales y las parasitemias se resume en el cuadro 4A. Además, se anota que la parasitemia se eliminó en 24 horas de tratamiento con $\mathrm{CQ}$ y $\mathrm{PQ}$ en $11 \%$ de los enfermos (24/210), cifra que subió a $66 \%$ en 48 horas (139/210) y a $99 \%$ en 72 horas; también se halló que la fiebre desapareció en 92\% (193/210) en 24 horas de tratamiento con $C Q$ y $P Q$, porcentaje que se mantuvo a las 48 horas e incrementó a $97 \%$ en 72 horas. El día 3 (24 horas después de terminar la toma de CQ), hubo siete pacientes con parasitemia pero sin fiebre y dos pacientes con fiebre que no tenían parásitos. Nuestros pacientes tomaron simultáneamente $\mathrm{CQ}$ y $P Q$, de tal manera que, a las 24 horas de haber terminado la CQ (día 3 del protocolo), los grupos con 105 y $210 \mathrm{mg}$ de PQ llevaban cuatro dosis (una por día) de PQ (15 mg/día), mientras que el grupo con $45 \mathrm{mg}$ ya había terminado de tomarla; en ese día 3 no hubo diferencias en el comportamiento temporal de la parasitemia asexual, la gametocitemia y la temperatura corporal entre los tres grupos, y tampoco se hallaron en el día 28 (todos los 210 pacientes estaban sin fiebre y sin parásitos). 
Cuadro 4. Evolución temporal de las parasitemias y los signos vitales.

A. Grupo total $(n=210)$

\begin{tabular}{lrrrrr}
\hline Variable (promedios diarios) & \multicolumn{5}{c}{ Día de seguimiento } \\
\cline { 2 - 6 } & $\mathbf{0}$ & $\mathbf{1}$ & $\mathbf{2}$ & $\mathbf{3}^{(1)}$ & $\mathbf{2 8}$ \\
Parasitemia asexual/ $\mu \mathrm{L}$ & 5955 & 363 & 23 & 2 & 0 \\
Gametocitos/ $\mu \mathrm{L}\left({ }^{(0)}\right.$ & 503 & 102 & 28 & 0 & 0 \\
${\text { Temperatura }{ }^{\circ} \mathrm{C}}^{(2)}$ & 37,3 & 36,6 & 36,3 & 36,4 & 36,6 \\
Fiebre subjetiva & 69,8 & 63,7 & 16,1 & 3,9 & 5,1 \\
Pulso/minuto & 85 & 77 & 73 & 71 & 73 \\
Frecuencia respiratoria/min & 22 & 20 & 20 & 20 & 20 \\
Tensión arterial sistólica mm Hg & 102 & 97 & 97 & 97 & 103 \\
Tensión arterial diastólica mm Hg & 64 & 64 & 63 & 62 & 66 \\
\hline
\end{tabular}

(0) El total de pacientes con medición de gametocitos es $n=47$.

(1) El día 3 (24 horas después de terminar la CQ), hubo 7 pacientes con parasitemia asexual, 4 con gametocitemia, 2 con fiebre; ninguno presentó simultáneamente fiebre y parasitemia; ver explicación en el texto.

(2) Porcentaje de pacientes con percepción de fiebre.

B. Grupo de pacientes con eliminación lenta de parásitos y fiebre $(n=9)^{(0)}$

\begin{tabular}{lccccc}
\hline Variable & N & Mínimo & Máximo & Media & D.E. \\
\hline Edad (años) & 9 & 17 & 41 & 23,33 & 7,14 \\
Peso (Kg) & 9 & 56 & 77 & 63,8 & 7,66 \\
Episodios paludismo ${ }^{(1)}$ & 3 & 1 & 2 & 1,33 & 0,58 \\
Días evolución enfermedad $_{\text {Parásitos asexuales } / \mu \mathrm{L}}$ & 9 & 2 & 8 & 4,33 & 2,1 \\
Gametocitos $/ \mu \mathrm{L}^{\text {Temperatura }}{ }^{\circ} \mathrm{C}$ & 9 & 200 & 20640 & 10538 & 7330 \\
\hline
\end{tabular}

(0) Características en el día 0 (pretratamiento) de los pacientes que el día 3 (24 horas tras finalizar CQ) aún tenían parasitemia $(n=7)$ o fiebre $(n=2)$; ninguno mostró ambos signos en el día 3 .

(1) Cantidad de episodios de paludismo en el último año.

Los nueve pacientes que el día 3 aún tenían parasitemia $(n=7)$ o fiebre $(n=2)$ presentaban las características mostradas en el cuadro 4B; los datos indican que antes de iniciar el tratamiento estos pacientes lentos para eliminar parásitos o fiebre tenían un conjunto de características (un perfil) diferente a las del grupo total de 201 sujetos; en efecto, estos nueve pacientes tenían menos edad, menos antecedentes de episodios palúdicos y menos días de evolución de la enfermedad que el grupo total, mientras que poseían mayores parasitemia asexual y sexual y mayor temperatura corporal (fiebre). No se hizo análisis con pruebas estadísticas de estas diferencias porque el escaso número de sujetos en el grupo con "curación lenta" impedía un examen apropiado.

\section{Efectos adversos}

Se registraron 696 eventos adversos; los más frecuentes (5\% o más del total de 696) fueron: prurito, 7,8\%, mareo, 7,2\%, náusea, 7,0\%, escalofrío, 6,7\%, sudoración, 6,5\%, fiebre, $6,4 \%$, dolor abdominal, $5,8 \%$, palidez conjuntival, $5,5 \%$, vómito, 5,4\%, diarrea, 5,3\%. Como puede concluirse, todos ellos son parte del cuadro básico de paludismo, excepto el prurito. Todos los demás eventos adversos registrados también se ajustan a los síntomas y signos maláricos.

La relación de los eventos adversos con el tratamiento antimalárico (CQ-PQ) se juzgó como nula en $48 \%$, como posible en $17 \%$, como poco probable en $13 \%$, probable en $13 \%$ y definitiva en 9\% (los más frecuentes de esos 62 fueron el prurito, $84 \%$, y las náuseas, $5 \%$ ). Menos de $1 \%$ de los eventos se valoró como grave, menos del $1 \%$ requirió hospitalización (un paciente con ictericia y coluria, caso considerado como malaria complicada, pero sin relación "definitiva" con el tratamiento) y $91 \%$ no requirió adoptar ninguna 
medida o intervención específica. Los eventos adversos desaparecieron totalmente en $93 \%$ de los pacientes al finalizar la observación, en 5\% persistía y en $2 \%$ no había dato. Cuanto mayor fue la duración del tratamiento con $P Q$, mayor fue la frecuencia de eventos adversos todavía presente: $16 \%$ con 3 días, $32 \%$ con 7 días y $51 \%$ con 14 días.

La clase y frecuencia de los eventos adversos fue estadísticamente igual entre los tres grupos (tomaron la misma cantidad de $C Q$ y dosis total diferente de $P Q$ ), de tal manera que el uso de $P Q$ durante 14 días no generó más eventos adversos que su empleo durante tres días únicamente.

\section{Discusión}

El cuadro clínico de la malaria por $P$. vivax no complicada encontrado en este trabajo en Turbo y El Bagre es en esencia similar al descrito por Echeverri y colaboradores para personas de $2 \mathrm{a}$ 75 años en Turbo (3). De igual manera, es similar al descrito recientemente por Duarte y asociados para pacientes brasileros (37). Se trata de un síndrome febril de cinco días de evolución que no tiene características patognomónicas, y en los que únicamente la demostración de parásitos del género Plasmodium permite una clasificación específica y, en consecuencia, un tratamiento específico.

Según se anotó, la frecuencia de síntomas fue estadísticamente mayor en El Bagre que en Turbo. Al respecto puede anotarse que el nivel endémico de paludismo en El Bagre es casi el doble que en Turbo (1998-2004: 47,46 por mil vs. 85,32 por mil, respectivamente). Según esos datos, que son similares en muchas otras épocas (más endemia en El Bagre que en Turbo), cabría esperar lo contrario de lo observado, es decir menor frecuencia e intensidad de los síntomas en El Bagre, suponiendo que la mayor endemia implica desarrollar inmunidad antimalárica más precoz y más fuerte.

La cantidad de pacientes seguidos durante 28 días para medir la respuesta terapéutica antimalárica con el esquema de $C Q$ y $P Q$ concuerda con lo esperado para conservar la representatividad de la muestra: el tamaño muestral calculado fue de
210 sujetos y en esa cantidad se midió la respuesta terapéutica antimalárica.

Cabe destacar que la eficacia de este tratamiento medida con criterios clínicos y parasitológicos fue de $100 \%$ con cualquiera de las tres dosis totales de PQ. Resulta claro que haber supuesto una diferencia de $15 \%$ en la frecuencia de falla entre los dos grupos extremos fue una sobrestimación derivada del único trabajo colombiano que dijo haber encontrado falla de una magnitud de $11 \%$ (9). Dada la muy elevada eficacia del tratamiento de $C Q$ y $P Q$, las futuras evaluaciones deberían suponer diferencias mucho menores entre los grupos, lo cual implica un mayor tamaño de muestra.

La curación de la totalidad de los pacientes con el tratamiento de $C Q$ y $P Q$ dadas en forma simultánea es de gran importancia desde el punto de vista de salud pública. Ya se dijo que para aceptar una infección por $P$. vivax como resistente a cloroquina deben seguirse criterios precisos, y que apenas hay dos informes en América $(23,24)$ que cumplen los criterios de Ruebush y colaboradores (2003) (24), lo que elimina los otros $(9,21,22)$, incluido el de Colombia (9), el cual corresponde a una evaluación de la $\mathrm{CQ}$ en soldados colombianos y es la única referencia a posible falla o resistencia de $P$. vivax a tal droga. Hay que resaltar que esos soldados recibieron sólo $\mathrm{CQ}$ (no tomaron $\mathrm{PQ}$ ), por lo que no puede hablarse de falla del tratamiento estándar, pues éste incluye la PQ. Además de las objeciones ya anotadas, la falla informada ( $11 \%$ ) se encontró en dos lugares muy diferentes de Colombia: Ios Llanos Orientales ( $n=16$; falla: $1 / 16)$ y Urabá $(n=11$; falla: $2 / 11)$. El grupo de personas de Urabá era muy reducido $(n=11)$ comparado con el del presente trabajo ( $n$ $=210$ ), lo que permite conclusiones más confiables en nuestro caso.

Hemos evaluado la eficacia de la CQ frente a $P$. vivax en varias ocasiones y siempre ha sido de 100\%. En 1998 se estudiaron pacientes en Turbo $(n=30)$ y en Zaragoza $(n=66)$, quienes recibieron el tratamiento estándar ( $C Q$ y luego $P Q$ ) y fueron seguidos durante 16 días (38); en 2001, se evaluaron 33 pacientes en Turbo con el protocolo OMS-2001 (18) y seguimiento de 28 días; se 
administró sólo $C Q$ en la forma estándar (la $P Q$ se aplicó únicamente a partir del día 28) (30). Entre 2003 y 2006 se ha evaluado la respuesta a CQ y a CQ más PQ en Turbo y El Bagre en decenas de pacientes, y siempre la eficacia ha sido de $100 \%$ (datos no publicados). Además, en otra región colombiana diferente a todas las anteriores (Cali, Valle del Cauca), se evaluó entre 1998 y 1999 la eficacia de la CQ en dosificación estándar en 44 pacientes con malaria por $P$. vivax, y se encontró respuesta adecuada en $100 \%$ con seguimiento de 28 días tras aplicar el protocolo de la OMS (39). En síntesis, todo indica que la eficacia del tratamiento de $C Q$ y $P Q$ para curar el ataque agudo del paludismo por $P$. vivax es total en diferentes regiones colombianas. Este hecho, sumado a otro que indica que el tratamiento amodiaquinasulfadoxina-pirimetamina eliminó la malaria por $P$. falciparum en $98 \%$ de los pacientes de Turbo, El Bagre y Zaragoza (40), demuestra que el grave problema de salud pública que constituye la malaria en Colombia no se debe a la falla de los tratamientos cuando se prescriben y usan adecuadamente ni a la resistencia de Plasmodium a ellos, como algunos lo afirman sin dar argumentos, hecho que hemos criticado $(1,2)$.

Ya se describió la evolución temporal de la fiebre y la parasitemia asexual y se dijo que en el día 3, $3 \%$ de los pacientes presentaba parásitos asexuales en sangre, pero cada uno con una cantidad inferior a $1 \%$ del valor inicial y además sin fiebre (temperatura axilar $>37,5^{\circ} \mathrm{C}$ ). De otra parte, los dos pacientes (1\%) con fiebre en ese día 3 estaban sin parasitemia. Es claro, en consecuencia, que en estos pacientes no se presentaron fallas terapéuticas, aunque ciertamente mostraron una menor capacidad de eliminar los parásitos o la fiebre, pero siempre en el "intervalo de lo normal". Llama la atención que este grupo de nueve pacientes con "lentitud" en la eliminación de los parásitos o la fiebre tiene características distintas de las observadas en el grupo total de 210 personas. Estos eran más jóvenes, tenían menos antecedentes de malaria $y$, en consecuencia, menos inmunidad específica (lo que concuerda con su juventud), y menos días de evolución de la enfermedad, pero presentaban mayores parasitemia asexual y sexual y mayor temperatura corporal (fiebre), lo cual tiene coherencia, pues el hecho de ser más jóvenes, presentar menos antecedentes de malaria y tener menor inmunidad implica mayor parasitemia y más fiebre.

Los efectos adversos observados fueron muy frecuentes, pero prácticamente todos corresponden a los síntomas y signos propios de la malaria, y su cantidad se debe a la sensibilidad de la definición empleada con el fin de no dejar pasar fenómenos inobservados. Solamente el prurito, que fue el efecto adverso más frecuente, podría considerarse relacionado con el tratamiento antimalárico y ha sido reconocido como asociado al uso del esquizonticida $C Q$ (35), aunque no con la $P Q(35,41)$. Los efectos adversos fueron leves en más de un $99 \%$, desaparecieron total y espontáneamente y no dejaron secuelas.

Se recuerda que hay informes que llaman la atención sobre la necesidad de explicar adecuadamente al paciente para qué es el tratamiento con $P Q$, cuáles son sus ventajas, y cuáles los posibles efectos adversos; uno de tales reportes encontró que la adherencia al tratamiento no fue menor con supervisión que sin ella, siempre que a este último grupo se le explicara y motivara apropiadamente para hacerse la terapia (41).

Es conveniente evaluar a fondo los efectos del suministro simultáneo de $C Q$ y $P Q$ en vez de aministrarlos en secuencia. Desde hace más de 50 años se sabe de los efectos de sinergismo entre los dos fármacos $(16,17)$ y ello se reiteró en 2005 (42) en un estudio en el que los autores encontraron que "la primaquina es un potente sinergizador de la actividad de la cloroquina contra $P$. falciparum cloroquino-resistente (...). La primaquina ejerce su actividad mediante bloqueo del transportador de resistencia a cloroquina de $P$. falciparum (PfCRT) y así aumenta la acumulación de cloroquina" (42). Agregan los autores que sus "datos sugieren que una nueva formulación de los dos antimaláricos, ya licenciada para uso en humanos, podría usarse para tratar parásitos cloroquino-resistentes" (42). Hay que advertir, por supuesto, que estos hallazgos corresponden a $P$. falciparum, pero en 1955 se informó que el fenómeno de potenciación 
de la actividad curativa de la $P Q$ contra $P$. vivax se incrementaba con el uso de $C Q$ o de quinina (16), aunque trabajos recientes no muestran tal efecto, como se plantea enseguida.

La actividad de la $P Q$ contra los estadios asexuales sanguíneos de Plasmodium se conoce desde hace más de 50 años; en 1953 (43) y en 1969 (44) se dieron a conocer informes en ese sentido, que fueron ratificados en 1994 (28) y en 2004 (45) por Pukrittayakamee y su grupo en Tailandia; sus trabajos demuestran que la $P Q$ dada como monoterapia $(0,25 \mathrm{mg} / \mathrm{kg} /$ día por 14 días) a pacientes con malaria por $P$. vivax es capaz de eliminar la fiebre con la misma eficacia y velocidad con que lo hacen la $C Q$ y la combinación de $C Q$ y $P Q$; también es eficaz para eliminar la parasitemia, pero más lentamente que esos otros dos tratamientos (33 horas vs. 24 horas); las recurrencias en el grupo de $P Q(n=4)$ fueron precoces (18 a 38 días), mientras que en el grupo de $\mathrm{CQ}(\mathrm{n}=4)$ fueron más tardías (42 a 51 días), lo mismo que en el grupo de $C Q$ más $P Q(n=2 ; 65$ a 97 días) (28). Como monoterapia, la $P Q$ es capaz de eliminar la parasitemia asexual, pero mucho más lentamente que la $C Q$, el artesunato y el artemeter, aunque bastante parecido a como lo hacen la mefloquina, la halofantrina y la quinina, y más rápido de lo que se logra con sulfadoxinapirimetamina; la $C Q$, con o sin $P Q$, eliminó bien la parasitemia $(28,45)$, con poco efecto adicional atribuible a la $P Q$, lo que sugiere ausencia de sinergismo. La tasa acumulada de curación a los 30 días fue baja y similar para artesunato, artemeter, halofantrina y quinina (es decir, hubo mucha recurrencia del tipo recrudescencia), mientras que fue de $100 \%$ para $C Q$ y mefloquina y alrededor de $95 \%$ para $P Q(45)$.

Por otra parte, según datos nuestros provenientes de estudios en desarrollo, el suministro simultáneo de $C Q$ y $P Q$ no aumenta la cantidad, la gravedad ni la duración de los efectos adversos. Los dos estudios de Tailandia también hallaron buena tolerancia a la $C Q$, la $P Q$ y la combinación de $C Q$ y $P Q$, con pocos efectos adversos y sin pruebas de hemólisis $(28,45)$. La aplicación concomitante importa por su mayor eficacia posible con igual impacto de los eventos adversos, y por el potencial que tiene de reducir el incumplimiento del tratamiento, sobre todo de la $P Q$, que se aplica durante 14 días después de haber tomado $\mathrm{CQ}$ por tres días, lo cual suma 17 días, un lapso relativamente prolongado, sobre todo si se considera que después de tres a cuatro días el paciente alcanza una gran mejoría, que puede ser estímulo para no continuar con el consumo de la $P Q$. Se requiere tener presente que la farmacocinética de la $P Q$ y de su principal metabolito (activo), la carboxi-primaquina, varían mucho y es posible que existan diferencias importantes entre grupos étnicos, lo que requiere investigación (46). La PQ es metabolizada en el hígado por las enzimas CYP1A2 y CYP2D6 del citocromo $\mathrm{P} 450$, mientras la $C Q$ lo es por las CYP2C8, CYP3A4 y CYP2D6; la PQ es un potente inhibidor de la actividad de la CYP3A4 (47).

En síntesis, nuestros datos y la interpretación que de ellos hacemos indican que la totalidad de los pacientes tratados con la administración simultánea de $C Q$ y $P Q$ respondió adecuadamente, presentando eliminación de la fiebre y la parasitemia en el tiempo y las cantidades esperadas según el protocolo de evaluación. En general, el uso simultáneo de los dos medicamentos no se asoció con eventos adversos graves y en $9 \%$ de los casos se consideró que había relación causal entre el evento aparecido y el tratamiento. La clase y frecuencia de eventos adversos fue similar con el uso de $P Q$ por 14 días o por tres días. Considerando la relación de sinergia entre $C Q$ y $P Q$, sugerimos que se adopte la administración concomitante de $C Q$ y $P Q$ para el tratamiento de la malaria por $P$. vivax no complicada. El esquema de $C Q$ y $P Q$ en la dosificación usual ( $\mathrm{mg} / \mathrm{kg}$, fracciones/día, días de aplicación) debe conservarse como la primera opción terapéutica y debe evaluarse en otras áreas. Debe examinarse el desempeño de este esquema terapéutico en menores de 14 años. Desde el punto de vista del tratamiento del ataque agudo, bastaría con dar la PQ por tres días en vez de 14 días, pero a esto debe agregarse la consideración de que en la malaria por $P$. vivax, la $P Q$ se suministra esencialmente para prevenir las recaídas, puesto que la CQ es suficiente para eliminar las formas sanguíneas sexuales y asexuales. Actualmente, estamos evaluando el 
problema de cuántos mg/día/kg y durante cuántos días debe administrarse la $P Q$ para la prevención adecuada de las recaídas.

\section{Agradecimientos}

A los directivos y personal de los hospitales locales de Turbo y El Bagre por su generosa y entusiasta ayuda.

A los pacientes por su participación en este trabajo que procura exclusivamente su bienestar.

A los profesionales del Grupo de Malaria que hicieron el trabajo de campo en El Bagre y Turbo.

\section{Conflicto de intereses}

Los investigadores no tienen ni han tenido vínculo o conflicto de ninguna clase con los productores o distribuidores de los medicamentos usados.

\section{Financiación}

Esta investigación fue financiada por la Dirección Seccional de Salud de Antioquia (DSSA) y la Universidad de Antioquia.

\section{Referencias}

1. Carmona-Fonseca J. La malaria en Colombia, Antioquia y las zonas de Urabá y Bajo Cauca: panorama para interpretar la falla terapéutica antimalárica. Parte 1. latreia $2003 ; 16: 299-318$

2. Carmona-Fonseca J. La malaria en Colombia, Antioquia y las zonas de Urabá y Bajo Cauca: panorama para interpretar la falla terapéutica antimalárica. Parte 2. Iatreia 2004;17:34-53.

3. Echeverri M, Tobón A, Álvarez G, Carmona J, Blair S. Clinical and laboratory findings of Plasmodium vivax malaria in Colombia, 2001. Rev Inst Med Trop São Paulo 2003;45:29-34.

4. Ríos-Orrego A, Álvarez-Castillo T, CarmonaFonseca J, Blair S. Evolución temporal de las plaquetas y los anticuerpos antiplaquetarios en pacientes de área endémica con malaria no complicada. An Med Interna 2005;22:561-8.

5. Blair S, Álvarez G, Campuzano G. Relación entre anemia y malaria en una población rural de Colombia. Boletín Dirección Malariología Saneamiento Ambiental 1997;37:7-13.

6. González L, Guzmán M, Carmona J, Lopera T, Blair S. Características clínico-epidemiológicas de 291 pacientes hospitalizados por malaria en Medellín (Colombia). Acta Med Colomb 2000; 25:163-70.

7. World Health Organization. The use of antimalarial drugs. Report of a WHO Informal Consultation. Geneva:
WHO; 2001. WHO/CDS/RBM/2001.33. [Consulta: febrero 12 de 2006]. Disponible en: http://www.who.int/ $\mathrm{malaria/cmc \_ upload/0/000/014/923/}$ use_of_antimalarials.pdf

8. Ministerio de Salud. Guía de atención clínica para el diagnóstico y tratamiento de la malaria. Bogotá: Minsalud; 1999.

9. Soto J, Toledo J, Gutierrez P, Luzz M, Llinas N, Cedeño $\mathbf{N}$, et al. Plasmodium vivax clinically resistant to chloroquine in Colombia. Am J Trop Med Hyg 2001;65:90-3.

10. Abdon NP, Pinto AY, da Silva Rdo S, de Souza JM. Assessment of the response to reduced treatment schemes for vivax malaria. Rev Soc Bras Med Trop 2001;34:343-8

11. da Silva RS, Pinto AY, Calvosa VS, de Souza JM. Esquemas terapêuticos encurtados para o tratamento de malária por Plasmodium vivax. Short course schemes for vivax malaria treatment. Rev Soc Bras Med Trop 2003;36:235-9.

12. Pinto AY, Ventura AM, Calvosa VS, Silva Filho MG, Santos MA, Silva RS, et al. Eficácia de quatro esquemas terapêuticos para malaria vivax em crianças. J Pediatr 1998;74:222-7.

13. Solari-Soto L, Soto-Tarazona A, Mendoza-Requena D, Llanos-Cuentas A. Ensayo clínico del tratamiento de la malaria vivax con esquema acortado de primaquina comparado con el esquema tradicional. Rev Soc Peru Med Interna 2002;15:196-9.

14. Pinto AY, Azevedo CH, da Silva JB, de Souza JM. Assessment of chloroquine single dose treatment of malaria due to Plasmodium vivax in Brazilian Amazon. Rev Inst Med Trop Sao Paulo 2003;45:327-31.

15. Carmona-Fonseca J. Profilaxis primaria con primaquina para el paludismo: revisión. Anuario de Enfermedades Infecciosas (Medellín) 2005;2:51-4.

16. Alving AS, Arnold J, Hockwald RS, Clayman CB, Dern RJ, Beutler E, et al. Potentiation of the curative action of primaquine in vivax malaria by quinine and chloroquine. J Lab Clin Med 1955;46:301-6.

17. Edgcomb JH, Arnold J, Yount EH Jr, Alving AS, Eichelberger L, Jeffery GM, et al. Primaquine, SN 13272, a new curative agent in vivax malaria: a preliminary report. J Natl Malar Soc 1950;9:285-92.

18. World Health Organization. A general guide for the assessment of therapeutic efficacy of chloroquine for vivax malaria (working draft). Version 3. Geneva: WHO; 2001.

19. Bergqvist Y, Domeij-Nyberg B. Distribution of chloroquine and its metabolite desethyl-chloroquine in human blood cells and its implication for the quantitative determination of these compounds in serum and plasma. J Chromatogr 1983;272:137-48. 
20. Whitby M. Drug resistant Plasmodium vivax malaria. $\mathrm{J}$ Antimicrob Chemother 1997;40:749-52.

21. Garavelli PL, Corti E. Chloroquine resistance in Plasmodium vivax: the first case in Brazil. Trans $\mathrm{R}$ Soc Trop Med Hyg 1992;86:128.

22. Alecrim M das C, Alecrim W, Macedo V. Plasmodium vivax resistance to chloroquine (R2) and mefloquine (R3) in Brazilian amazon region. Rev Soc Bras Med Trop 1999;32:67-8.

23. Phillips EJ, Keystone JS, Kain KC. Failure of combined chloroquine and high dose primaquine therapy for Plasmodium vivax malaria acquired in Guyana, South America. Clin Infect Dis 1996;23:1171-3.

24. Ruebush TK 2nd, Zegarra J, Cairo J, Andersen EM, Green M, Pillai DR, et al. Chloroquine-resistant Plasmodium vivax malaria in Peru. Am J Trop Med Hyg 2003;69:548-52.

25. Baird JK, Leksana B, Masbar S, Fryauff DJ, Sutanihardja MA, Suradi L, et al. Diagnosis of resistance to chloroquine by Plasmodium vivax: timing of recurrence and whole blood chloroquine levels. Am J Trop Med Hyg 1997;56:621-6.

26. Bruce-Chwatt LJ. Essential malariology. London: William Heinemann Medical Books Ltd; 1980. p.179.

27. Mollinedo S, Mollinedo R. La malaria en Bolivia. La Paz: Ministerio de Salud y Previsión Social; 2000.p.63.

28. Pukkrittayakamee S, Vanijanonta S, Chantra A, Clemens R, White NJ. Blood stage antimalarial efficacy of primaquine in Plasmodium vivax malaria. J Infect Dis 1994;169:932-5.

29. World Health Organization. Guidelines for the treatment of malaria. Geneva: WHO; 2006. WHO/HTM/MAL/ 2006.1108.

30. Blair S, Tobón A, Echeverri M, Álvarez G, CarmonaFonseca J. Adecuada respuesta clínica y parasitológica de Plasmodium vivax a la cloroquina en Colombia (Turbo, Antioquia), 2001. Infectio 2002;6:216.

31. Carmona-Fonseca J. Malaria, desnutrición y parasitosis intestinal en los niños colombianos: interrelaciones. latreia 2004;17:354-69.

32. Bloland PB. Drug resistance in malaria. Geneve: WHO; 2001. WHO/CDS/CSR/DRS/2001.4

33. López-Antuñano FJ. Diagnóstico microscópico de los parásitos de la malaria en la sangre. En: LopezAntuñano FJ, Schmunis G, editores. Diagnostico de malaria. Volumen 512. Washington: OPS-OMS; 1988. p. $39-50$

34. Organización Panamericana de la Salud, Organización Mundial de la Salud. Evaluación de la eficacia terapéutica de los medicamentos para el tratamiento del paludismo por Plasmodium falciparum sin complicaciones en las Américas. Washington: OPS-
OMS; 1998. OPS/HCP/HCT/113/98.

35. World Health Organization WHO. Assessment and monitoring of antimalarial drug efficacy for the treatment of uncomplicated falciparum malaria. Geneva; WHO, 2003. Documento WHO/CDS/CSR/RMB.

36. Segura O, Maldonado CE. Las reacciones adversas a medicamentos: una aproximación desde el punto de vista económico. Biomédica 2003;23:401-7.

37. Duarte EC, Pang LW, Ribeiro LC, Fontes CJ. Association of subtherapeutic dosages of a standard drug regimen with failures in preventing relapses of vivax malaria. Am J Trop Med Hyg 2001;65:471-6.

38. Lacharme L, Carmona-Fonseca J, Tobón A, Blair S. Respuesta de $P$. vivax al esquema terapéutico cloroquina-primaquina en Zaragoza y Turbo, Colombia, 1998. Infectio 1998;2:90-4.

39. Castillo CM, Osorio LE, Palma GI. Assessment of therapeutic response of Plasmodium vivax and Plasmodium falciparum to chloroquine in a malaria transmission free area in Colombia. Mem Inst Oswaldo Cruz 2002;97:559-62.

40. Carmona-Fonseca J, Tobón A, Álvarez G, Blair S. El tratamiento amodiaquina-sulfadoxina-pirimetamina tiene eficacia del $98 \%$ para la malaria falciparum no complicada (Antioquia, Colombia; 2003). latreia 2005; 18:5-26.

41. Leslie T, Rab MA, Ahmadzai H, Durrani N, Fayaz M, Kolaczinski J, et al. Compliance with 14-day primaquine therapy for radical cure of vivax malaria-a randomized placebo-controlled trial comparing unsupervised with supervised treatment. Trans $\mathrm{R}$ Soc Trop Med Hyg 2004; 98:168-73.

42. Bray PG, Deed S, Fox E, Kalkanidis M, Mungthin M, Deady LW, et al. Primaquine synergises the activity of chloroquine against chloroquine-resistant $P$. falciparum. Biochem Pharmacol 2005;70:1158-66.

43. Cooper WC, Myatt AV, Hernandez ZT, Jeffrey GM, Coatney GR. Studies in human malaria. XXXI. Comparison of primaquine, isopentaquine, SN-3883, and pamaquine as curative agents against Chesson strain vivax malaria. Am J Trop Med Hyg 1953;2:949 57.

44. Schimidt LH. Chemotherapy of the drug-resistant malarias. Annu Rev Microbiol 1969;23:427-54.

45. Pukrittayakamee S, Imwong M, Looareesuwan S, White NJ. Therapeutic response to antimalarial and antibacterial drugs in vivax malaria. Acta Trop 2004;89:351-6.

46. Kim YR, Kuh HJ, Kim MY, Kim YS, Chung WC, Kim SI et al. Pharmacokinetics of primaquine and carboxyprimaquine in Korean patients with vivax malaria. Arch Pharm Res 2004;27:576-80.

47. Guzmán V, Carmona-Fonseca J. El citocromo P-450 y la respuesta terapéutica antimalárica. Rev Panam Salud Publica 2006;19:9-22. 